Research Article

\title{
Echocardiographic Features of Patients with Coronary Heart Disease and Angina Pectoris under Deep Learning Algorithms
}

\author{
Xianjing Han $(\mathbb{D})$ and Guoxin Liang \\ Department of Ultrasonography, The First Affiliated Hospital of Qiqihar Medical College, Qiqihar 161041, Heilongjiang, China \\ Correspondence should be addressed to Guoxin Liang; liangguoxin@qmu.edu.cn
}

Received 22 September 2021; Revised 24 October 2021; Accepted 25 October 2021; Published 13 November 2021

Academic Editor: M Pallikonda Rajasekaran

Copyright (c) 2021 Xianjing Han and Guoxin Liang. This is an open access article distributed under the Creative Commons Attribution License, which permits unrestricted use, distribution, and reproduction in any medium, provided the original work is properly cited.

\begin{abstract}
Based on the VGG19-fully convolutional network (FCN) (VGG19-FCN) and U-Net model in the deep learning algorithms, the left ventricle in the ultrasonic cardiogram was segmented automatically. In addition, this study evaluated the value of ultrasonic cardiogram features after segmentation by the optimized algorithm in diagnosing patients with coronary heart disease (CHD) and angina pectorisody; patients with arrhythmia; and pa. In this study, 30 patients with confirmed CHD and 30 normal people without CHD from the same hospital in a certain area were selected as the research objects. Firstly, the VGG19-FCN and U-Net model algorithms were selected to automatically segment the left ventricular part of the apical four-chamber static image, which was realized through the weights of the fine-tune basic model algorithm. Subsequently, the experimental subjects were divided into a normal group and a CHD group, and the data were obtained through the ultrasonic cardiogram feature analysis of automatic segmentation by the algorithm. The differences in the ejection fraction (EF), left ventricular fractional shortening (FS), and E/A values (in early and late of the diastolic phase) of the left ventricle for patients in the two groups were compared. In addition, the ultrasonic cardiogram left ventricular segmentation results of normal people and patients with CHD were compared. A comprehensive analysis suggested that the U-Net model was more suitable for the practical application of automatic ultrasonic cardiogram segmentation. According to the analyzed data results, the global systolic function parameters (EF, FS, and E/A values) of the left ventricle for patients showed statistically obvious differences $(P<0.05)$. In summary, deep learning algorithms can effectively improve the efficiency of ultrasonic cardiogram left ventricular segmentation, show a great role in the diagnosis of CHD patients, and provide a reliable theoretical basis and foundation research on the subsequent CHD imaging diagnosis. The comprehensive analysis showed that the U-Net model was more suitable for the practical application of echocardiographic automatic segmentation, and this study can effectively improve the efficiency of echocardiographic left ventricular segmentation, which played an important role in the diagnosis of coronary heart disease, providing a reliable theoretical basis and foundation for subsequent CHD imaging research.
\end{abstract}

\section{Introduction}

With the increasing development of the society and the gradual improvement of people's living standards, cardiovascular disease has gradually become a high-risk disease, of which CHD is a typical representative of cardiovascular disease. Relevant data show that the number of CHD patients in China in 2018 was approximately 11 million, and the morbidity and mortality rates have gradually increased [1]. CHD is a CVD mainly caused by the hardening of coronary arteries. It is clinically classified into five types: occult CHD, angina pectoris, myocardial infarction, ischemic heart disease
(IHD), and sudden death [2, 3]. The patient's general symptoms are typical chest pain and sudden death, and the chest pain is mostly paroxysmal colic or squeezing pain. Approximately one-third of the patients experienced sudden death with CHD for the first time. Therefore, it is extremely important to evaluate and diagnose CHD correctly and efficiently. Because of the particularity of the left ventricle, it is generally selected to measure the left ventricle to reflect cardiac function in clinical practice.

At present, the commonly used CHD examination methods include radionuclide myocardial imaging, CT, ultrasonic cardiogram, and cardiac magnetic resonance 
imaging (MRI) $[4,5]$. These are all imaging techniques used to evaluate cardiac function. Ultrasonic cardiogram is currently the most commonly used noninvasive examination method for $\mathrm{CHD}$. It can perform a more comprehensive examination of the shape, structure, and left ventricular function of the heart. In addition to CHD, it also has important diagnostic value for thrombosis in the heart cavity, heart rupture, and ventricular aneurysm [6, 7], but its shortcomings are also obvious. The accuracy of the ultrasonic cardiogram currently used clinically is closely related to the experience of the examiner and depends on the prior knowledge of the person, but the results of the examination are too dependent on the examiner's technical experience due to differences in the level of examiners [8], which will cause a certain degree of missed diagnosis and misdiagnosis. Therefore, nowadays, researchers all over the world have begun to use deep learning algorithms to optimize the segmentation of the ultrasonic cardiogram so that the diagnosis result does not depend too much on the level and experience of the examiner, which can effectively improve the data accuracy of the ultrasonic cardiogram $[9,10]$.

Deep learning requires datasets to train models. However, it is difficult to obtain data. For a certain object, there are no enough data for training. In this case, it often takes time, money, and, most importantly, hardware equipment. Therefore, it needs a more systematic method to collect data. In addition, in the process of data labeling, it is not enough to rely solely on developers/engineers. This requires a large number of professionals with professional knowledge and experience to classify relevant images, especially for highly specialized fields such as medical diagnosis. Another key point is to provide education about general images in classic convolutional neural networks (CNNs) through class labels. However, there are some issues that require an understanding of the locality or location of pixel-based methods. In fields that require sensitive methods (such as biomedicine or national defense), class information for each pixel is required.

$\mathrm{U}-\mathrm{Net}$ is more successful in architecture design and other pixel-based image segmentation using the $\mathrm{CNN}$, and it is even more effective for images with limited datasets.

One of the three main methods of deep learning is the convolutional neural network (CNN). VGG19, FCN, and U-Net models are all algorithms that use the FCN for image segmentation. Deep learning technology is used in the segmentation processing of medical images, which can greatly improve the separation effect of the left ventricular region $[11,12]$. The FCN optimizes the fully connected layer in the traditional $\mathrm{CNN}$ to a convolutional layer and reduces the connection parameters of neurons through local links and weight sharing, reducing the complexity of calculations and improving the efficiency of segmentation. Clinical ultrasonic cardiogram parameter measurement generally requires manual marking of the region of interest and generally requires doctors to make judgments based on clinical experience, resulting in subjective errors. Therefore, the automatic segmentation of ultrasonic cardiogram images can be realized through the deep learning algorithm, which effectively improves the accuracy and repeatability of the parameter measurement.
Therefore, a model for ultrasonic cardiogram segmentation was trained based on deep learning algorithms, and the optimized ultrasonic cardiogram was applied to evaluate the diagnosis of CHD patients, and the differences in the evaluation of the overall functional parameters of the left ventricle of $\mathrm{CHD}$ patients were explored in this study.

\section{Materials and Methods}

2.1. Research Objects. 30 patients who were diagnosed with $\mathrm{CHD}$ and 30 normal patients who were excluded from CHD who were treated in the hospital were selected as the research objects in this study. This study had been approved by the Ethics Committee of the hospital, and the patient and his family members agreed to sign relevant informed consent.

The inclusion criteria were defined as follows: patients with coronary artery stenosis of $50 \%-70 \%$; patients with typical CHD angina pectoris but no evidence of coronary embolism or cardiomyopathy; patients who were 40 years old for males and over 45 years old for females with obvious ischemic electrocardiograph (ECG) manifestations at rest; and patients with two or more of the following diseases: hypertension, hypercholesterolemia, and diabetes.

The exclusion criteria were defined as follows: patients with coronary artery stenosis $<50 \%$ or $>70 \%$; patients whose age was less than 40 years; people with no symptom of $\mathrm{CHD}$ angina; patients with an implanted metal item in the body; patients with arrhythmia; and patients who suffered from claustrophobia.

2.2. The Ultrasonic Cardiogram Scan. The color ultrasound was applied, transthoracic ultrasound was equipped with an $S$ probe, and the frequency was $2.5-4 \mathrm{MHz}$. The patient had to adopt a left decubitus position and routinely explore the parasternal left ventricular long-axis view, left ventricular short-axis view, and apical four-chamber view. The global systolic function parameters (EF, FS, and E/A values) were obtained by applying an M-type ultrasonic cardiogram, which should be measured for three consecutive times to calculate the average value.

\subsection{Ultrasonic Cardiogram Segmentation Based on VGG19-} FCN. In this study, the VGG19-FCN model was adopted to achieve the segmentation of the ultrasonic cardiogram. The VGG19 model in Figure 1 is the backbone network, and the final network structure given in Figure 2 can be obtained by removing its full link layer and adding the sampling layer. Since the entire network structure only had the convolutional layer and the pooling layer after the full link layer was removed, this network structure was named VGG19-FCN.

Building a fully convolutional neural network model for left ventricular segmentation was mainly divided into two stages: training and testing. Firstly, the classification network was pretrained on VGG19, as shown in Figure 3. Then, the fully connected layer was removed and the sampling layer was added, and finally, it was transformed into a FCN for the next step of training. It was necessary to train the basic model VGG19-FCN/A, which was used for the automatic 


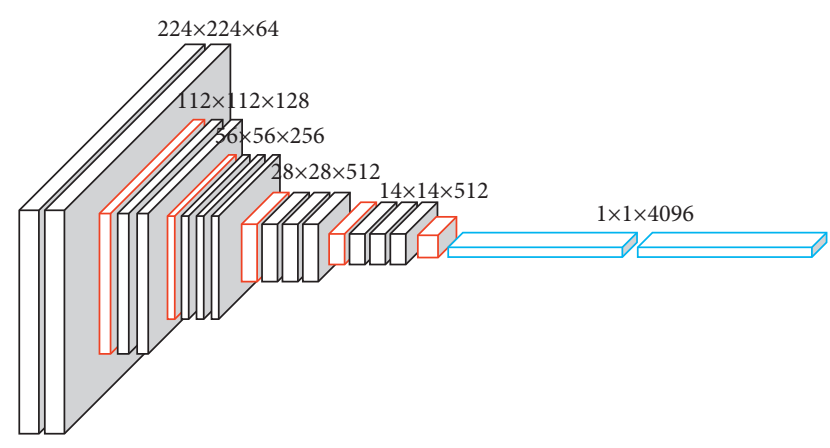

Convolution + ReLU

Max pooling

Fully connected + ReLU

FIGURE 1: Diagram of the VGG19 network structure.

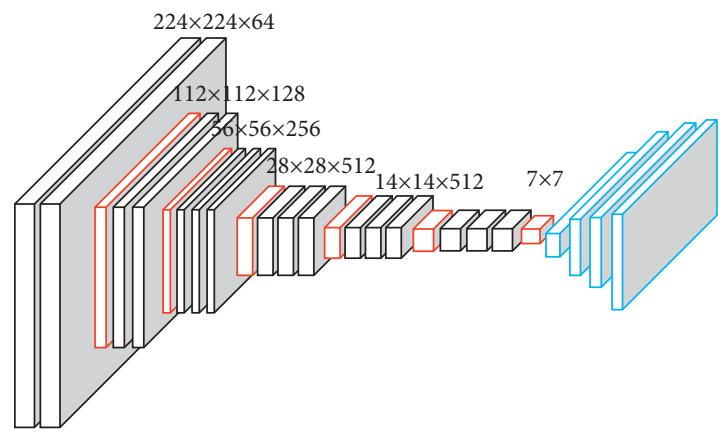

$$
\begin{aligned}
& \square \text { Convolution + ReLU } \\
& \square \text { Max pooling } \\
& \square \text { Fully connected + ReLU }
\end{aligned}
$$

Figure 2: Diagram of the VGG19-FCN network structure.

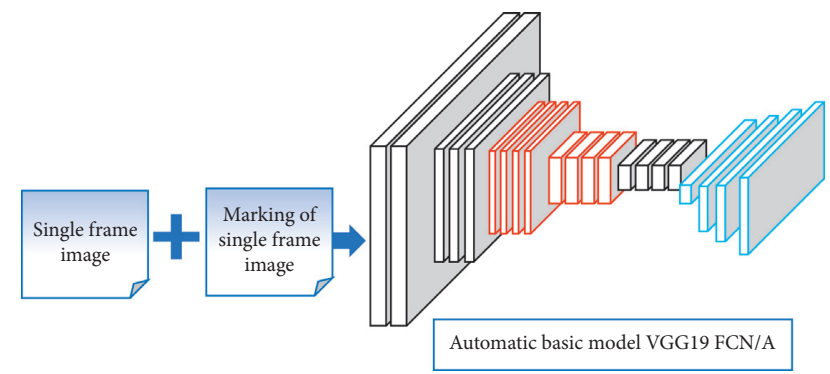

FIGURE 3: Training of the automatic basic VGG19-FCN/A model.

segmentation of ultrasonic static images of the left ventricle, and further conduct semisupervised learning for images with unclear boundaries [13]. Then, a new segmentation model VGG19-FCN/B was obtained according to the result of fine-tuning the weight of VGG19-FCN/A.

In this study, an algorithm based on the Dice similarity coefficient (DSC) was adopted to analyze all the data in the experiment, and the difference between images obtained by automatic segmentation and manual labeling was calculated. DSC is a function used to measure the similarity between functions $[14,15]$, and it could be calculated using the following equation:

$$
\mathrm{DSC}=\frac{2 \times|\mathrm{TS} \cap \mathrm{FP}|}{\mathrm{TS}+\mathrm{FP}} .
$$

In the above equation, TS represents the segmentation result obtained by the algorithm, and FP refers to the segmentation result of manual marking. When the value of DSC was closer to 1 , the automatic segmentation effect of the algorithm was better. When the value of DSC approached to zero, it indicated that the calculation ability of the algorithm was wrong. The calculation equation of mean pixel accuracy (MPA) was given as follows:

$$
\mathrm{MPA}=\left(1-\frac{|\mathrm{FP}-\mathrm{TS}|}{\mathrm{FP}}\right) .
$$

In equation (2), $|\mathrm{FP}-\mathrm{TS}|$ represents the number of pixels that were not correctly segmented, and MPA refers to the ratio of the number of pixels correctly distinguished to the number of manual markings. The larger the value of MPA, the better the result of algorithmic segmentation.

The equation of the intersection-over-union (IOU) metric is shown as follows:

$$
\mathrm{IOU}=\frac{\mathrm{FP} \cap \mathrm{TS}}{\mathrm{FP} \cup \mathrm{TS}} .
$$

IOU in the above equation represented the ratio between the intersection and union of the left ventricular result obtained by the algorithm and the result obtained by the manual marking. The higher the IOU value, the better the algorithm's segmentation effect.

2.4. Ultrasonic Cardiogram Segmentation Based on U-Net. Unlike the previous method, the VGG19-FCN required more parameters and was relatively inefficient, so a simplified U-Net was selected in this study to segment the ultrasonic cardiogram of the left ventricle. As shown in Figure 4, the U-Net model consisted of two paths, namely, the contraction path and the expansion path. U-Net can directly append the features corresponding to the contraction layer to the expansion layer so that the features of the image will not be lost and merge with the features of the expansion layer to output the segmented image. Because the $\mathrm{U}-\mathrm{Net}$ model is sensitive to the initial value, a specification layer after each layer of the U-Net was added in this study, which reduced the sensitivity of the network to the initial value and speeded up the convergence of the model.

The operating environment of the U-Net algorithm in the article was Python 3.6 (OpenCV-Python + 1 G memory + Windows XP).

Building a fully convolutional neural network, the U-Net model for left ventricular segmentation was mainly divided into two stages: training and testing. As shown in Figure 5, the U-Net model was trained first and applied to the basic model U-Net/A for the automatic segmentation of 


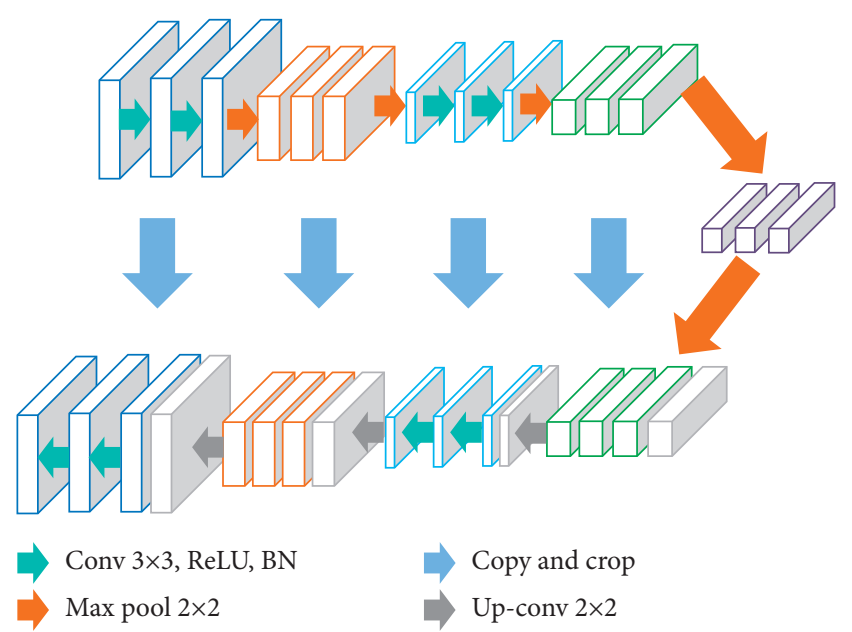

Figure 4: Diagram of the U-Net structure.

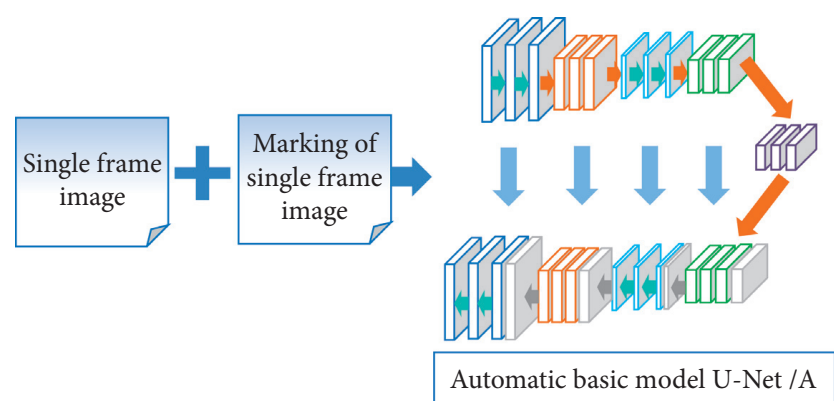

FIGURE 5: Training of the automatic basic U-Net/A model.

ultrasonic static images of the left ventricle. Semisupervised learning was further carried out for images with unclear boundaries, and then a new segmentation model U-Net/B was obtained based on the weight of fine-tune U-Net/A. Finally, the segmentation of the left ventricle in the ultrasonic cardiogram was achieved.

2.5. Statistical Analysis. SPSS 19.0 software was applied for statistical analysis. The measurement data conforming to the normal distribution were represented by the mean\pm standard deviation, and the comparison of differences between groups was analyzed by the independent sample $t$ test. The measurement data that did not conform to the normal distribution were represented by the median value and the four-point position, and the comparison of differences between groups was analyzed by the nonparametric rank-sum test. The enumeration data were expressed by $n$ (\%), and the comparison of differences between groups was analyzed by the chi-square test. $P<0.05$ indicated that the difference was statistically significant.

\section{Results}

3.1. Segmentation Effect of the VGG19-FCN Model. According to the VGG19-FCN/A, the segmentation result of the ultrasonic cardiogram left ventricle is shown in Figure 6.
The result of automatic segmentation according to the image was evaluated as $\mathrm{DSC}=0.9179, \quad \mathrm{MPA}=0.9209$, and $\mathrm{IOU}=0.8500$. For images with low signal-to-noise ratio and endocardial deletion, the segmentation results of the VGG19-FCN/A algorithm were not accurate. At this time, the VGG19-FCN/B algorithm with fine-tune weights was tested for the ultrasonic cardiogram left ventricular segmentation, and the results are shown in Figure 6. The result of automatic segmentation according to the image was evaluated as $\mathrm{DSC}=0.9626, \quad \mathrm{MPA}=0.9676$, and $\mathrm{IOU}=0.9155$. Compared with the VGG19-FCN/A algorithm, the VGG19-FCN/B algorithm required a certain amount of manual interaction, but the segmentation result of the ultrasonic cardiogram was closer to the result of the doctor's manual marking.

As illustrated in Figure 7, VGG19-FCN/B showed higher accuracy in segmenting the left ventricle compared to VGG19-FCN/A. The segmentation analysis data DSC, MPA, and IOU of the left ventricle in the ultrasonic cardiogram had increased by $0.0447,0.0467$, and 0.0655 , respectively.

3.2. Segmentation Effect of the U-Net Model. According to the U-Net/A, the segmentation result of the ultrasonic cardiogram of the left ventricle is shown in Figure 8. The result of automatic segmentation according to the image was evaluated as $\mathrm{DSC}=0.9152, \mathrm{MPA}=0.9229$, and $\mathrm{IOU}=0.8450$. Therefore, the segmentation results of the VGG19-FCN/A algorithm were not accurate. At this time, the U-Net/A algorithm was tested for the ultrasonic cardiogram left ventricular segmentation, and the results are shown in Figure 8 . The result of automatic segmentation according to the image was evaluated as $\mathrm{DSC}=0.9446, \mathrm{MPA}=0.9352$, and IOU $=0.8911$. Compared with the U-Net/A algorithm, the $\mathrm{U}-\mathrm{Net} / \mathrm{B}$ algorithm required a certain amount of manual interaction, but the segmentation result of the ultrasonic cardiogram was closer to the result of the doctor's manual marking.

The results shown in Figure 9 suggested that the U-Net/B showed higher accuracy than U-Net/A for segmenting the left ventricle. The segmentation analysis data DSC, MPA, and IOU of the left ventricle in the ultrasonic cardiogram had increased by $0.0294,0.0123$, and 0.0461 , respectively.

3.3. Comparison on Segmentation Effects between U-Net and VGG19-FCN Models. The segmentation accuracy of the two network model algorithms is compared, and the results are shown in Figure 10. On the whole, VGG19-FCN/A showed a higher accuracy rate than U-Net/A, and the accuracy rate of VGG19-FCN/B was also slightly higher than that of U-Net/ $B$. The amount of parameters required by the two network models was compared. The VGG19-FCN was modified from VGG19; so far, more parameter data were required than the $\mathrm{U}-\mathrm{Net}$ model. When these two models were applied and run, the more parameter data were required, the greater the amount of calculation is and the longer the calculation time is. The parameter data required by the VGG19-FCN were 360 times those of the U-Net model, but the result of the segmentation of the two was not different visibly. The U-Net 


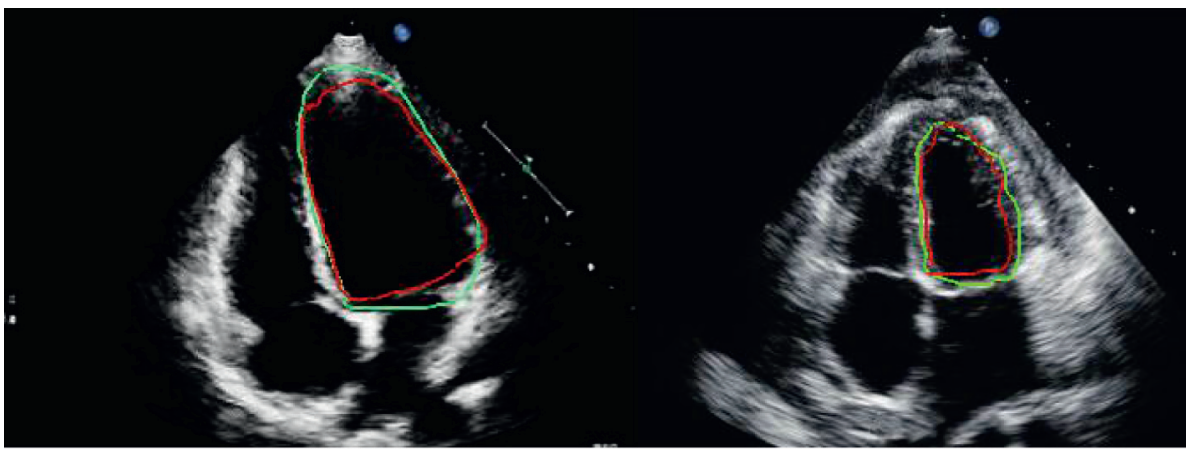

FIGURE 6: VGG19-FCN/A segmentation result of the ultrasonic cardiogram of the left ventricle. Note. The area manually marked was a green outline, and the automatic segmentation of the algorithm was a red outline.

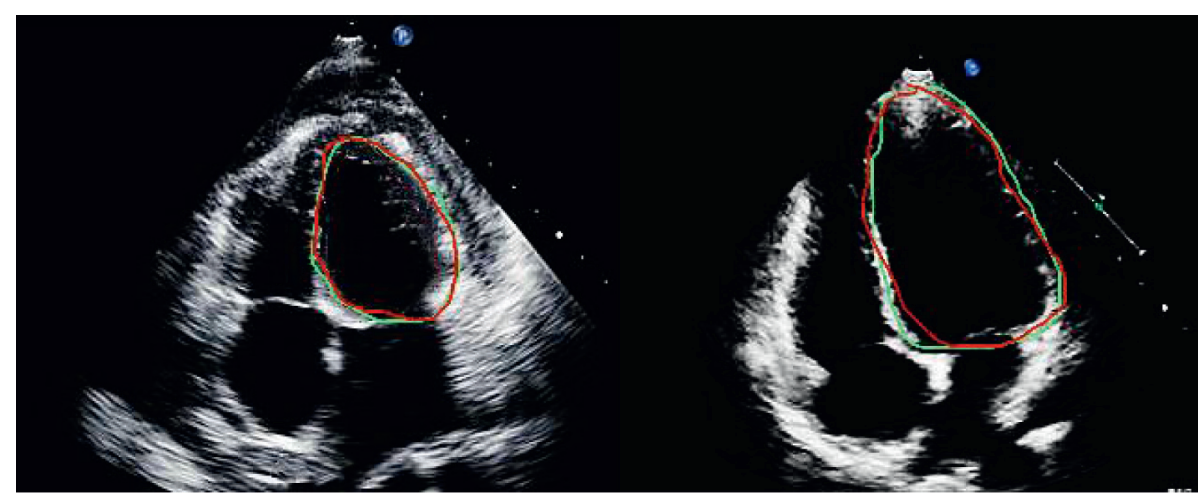

FIgURE 7: The segmentation result of the ultrasonic cardiogram of the left ventricle using VGG19-FCN/B. Note. The area manually marked was a green outline, and the automatic segmentation of the algorithm was a red outline.

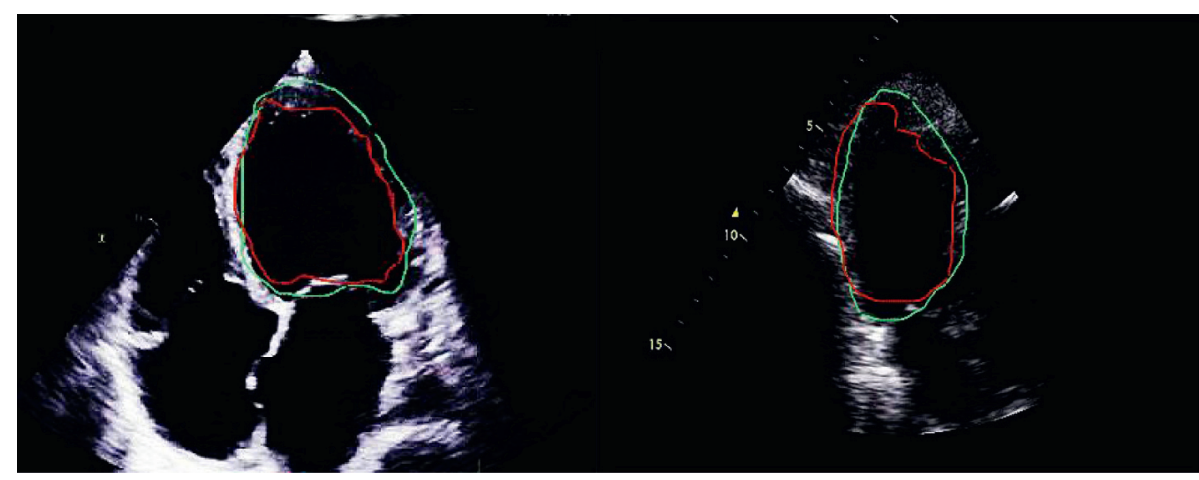

Figure 8: The segmentation results of the ultrasonic cardiogram of the left ventricle using the U-Net/A model. Note. The area manually marked was a green outline, and the automatic segmentation of the algorithm was a red outline.

model fully could meet the requirements for segmentation accuracy. There was a huge difference in the segmentation time between the two.

The running time of the VGG19-FCN was 13 times that of the U-Net model. Comprehensive analysis showed that the U-Net model was more suitable for the practical application of ultrasonic cardiogram automatic segmentation.

3.4. Comparison on Basic Conditions between the Normal Group and CHD Group. The basic information of the two groups of observation objects was collected and compared, as shown in Figure 11. In contrast to the normal group and the CHD group, there was no great difference in statistics $(P<0.05)$.

3.5. Comparison on Left Ventricular Function Parameters between the Normal Group and CHD Group by the Ultrasonic Cardiogram. The left ventricular ultrasonic cardiogram automatically segmented by the U-Net model was adopted to determine the overall left ventricular functional 


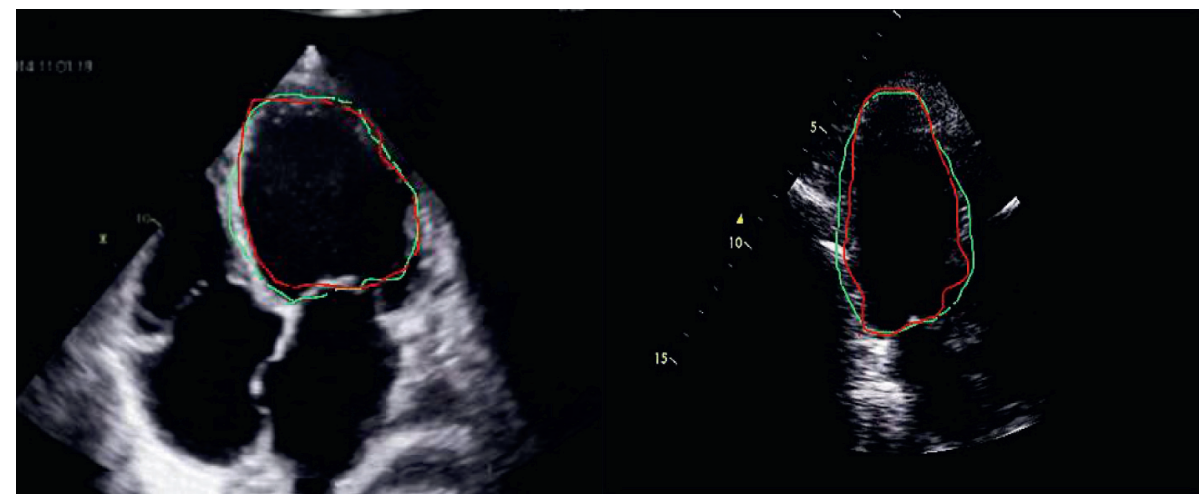

Figure 9: The segmentation result of the ultrasonic cardiogram of the left ventricle using the U-Net/B model. Note. The area manually marked was a green outline, and the automatic segmentation of the algorithm was a red outline.

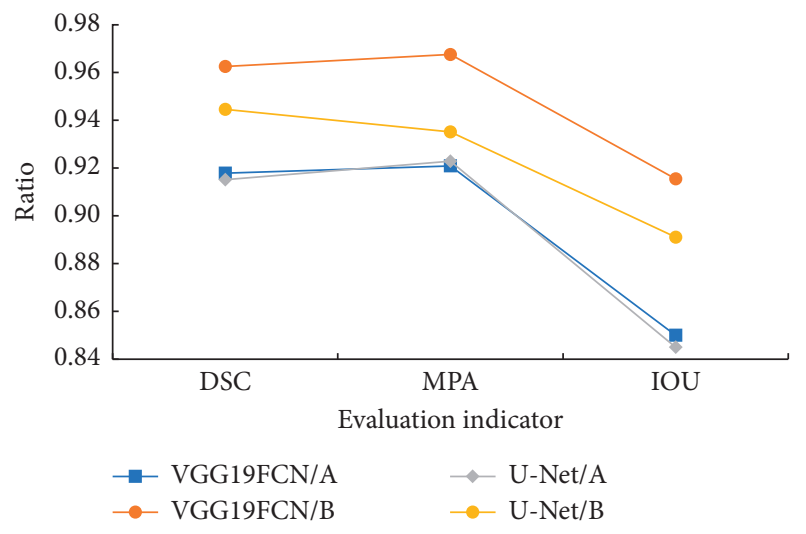

FIGURE 10: Comparison of segmentation effects between the U-Net and VGG19-FCN models.

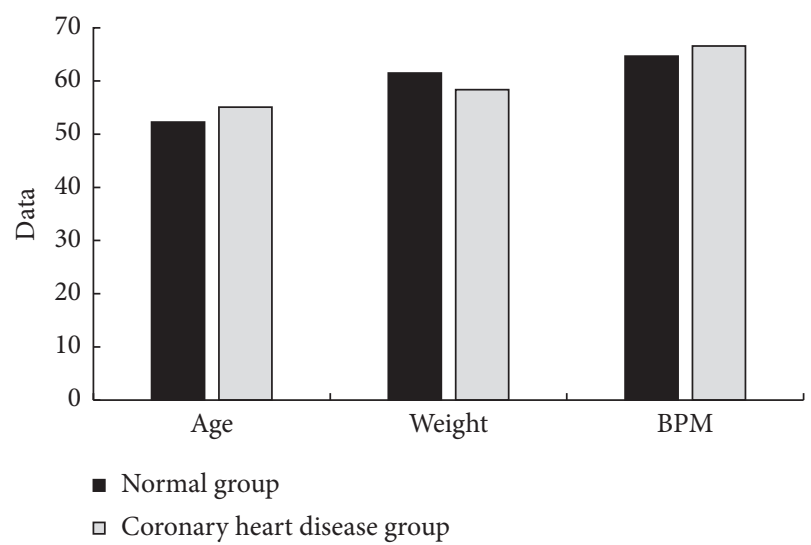

FIGURE 11: Comparison of the basic conditions of the two groups of observation objects. Note. BMP referred to the heart rate of the patient.

parameters of the two groups of observation objects, and the results are shown in Figure 12. The ultrasonic cardiogram was applied to measure the parameters of patients in the normal group and the CHD group, and they all met the normal distribution after the normality test. The

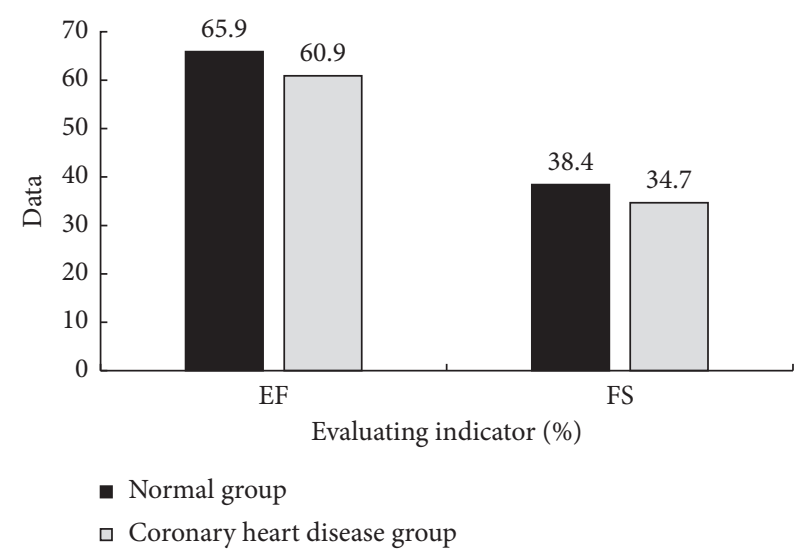

FIGURE 12: Comparison on left ventricular functional parameters of the two groups of observation objects.

homogeneity of the variance test did not meet the homogeneity of variance, so the corrected $t$-test for uneven variance was used. The $t$-test results of the two groups of data showed that the two groups of left ventricular global systolic function parameters (EF, FS value, and E/A values) showed statistically obvious differences $(P<0.05)$. Therefore, the left ventricular systolic function parameters EF, FS, and E/A values of the CHD group were lower than those of the normal group. According to the results, the left ventricular global systolic function of most patients in the CHD group in this experiment was still within the normal range; all patients in the CHD group had abnormal left ventricular global systolic function E/A, and the normal group observed objects were basically within the normal range.

\section{Discussion}

$\mathrm{CHD}$ is the full name of coronary atherosclerosis. The general cause is that the coronary arteries become narrow so that the blood and oxygen supply to the heart decreases. At this time, the heart cannot get more oxygenated blood. People with mild symptoms usually only experience physical discomfort or chest pain after exercise. The main part is the middle and upper part of the sternum and then radiates to the left shoulder, the inner left side of the upper arm, and the 
neck. Accompanied by pain, symptoms such as shortness of breath, tooth pain, cold sweats, nausea, and vomiting of varying severity may occur $[3,16]$.

According to relevant data, CVD accounted for $30 \%$ of deaths in the world in 2008, of which CHD accounted for $13 \%$. In 2012, the number of cardiovascular disease patients in China has reached 300 million, of which the annual death toll has reached 3.5 million $[17,18]$. Although the global CHD fatality rate has declined to a certain extent with the development of medical technology in recent years, with the continuous growth of the population and the development of an aging society, the absolute number of patients who died of CHD is still increasing. Among them, early detection and early diagnosis are the key to effective prevention and treatment of myocardial infarction, reducing the rate of sudden CHD death and controlling the development of CHD. Therefore, the research on the prevention and diagnosis of CHD is of great significance.

There are many commonly used methods for diagnosing CHD. Noninvasive imaging examination is the main method for diagnosing CHD, including ultrasonic cardiogram, electrocardiogram, cardiac magnetic resonance, nuclear myocardial imaging, coronary CT, and other imaging examination methods. The ultrasonic cardiogram has been used as the preferred method in the evaluation of cardiac structure and function because of its advantages of nonradiation, high resolution, and noninvasiveness [19]. In clinical applications, the relevant parameter data obtained from the ultrasonic cardiogram can help doctors diagnose $\mathrm{CHD}$, but the diagnosis efficiency is low and the repetition rate is extremely poor due to the need for human judgment and operation in the process. Therefore, this study aimed to explore the automatic segmentation algorithm of the ultrasonic cardiogram. The ultrasonic cardiogram segmentation of the left ventricle of the new organ is an important step in the diagnosis of cardiovascular diseases because the ultrasonic cardiogram has the problem of low signal-to-noise ratio and missing endocardium $[20,21]$. In this study, the experimental subjects were divided into the normal group and CHD group, and the data were obtained through the echocardiographic feature analysis of automatic segmentation by the algorithm. The differences in the left ventricular $\mathrm{EF}, \mathrm{FS}$, and E/A values between the two groups were compared. In addition, the results of echocardiographic left ventricular segmentation of normal people and patients with CHD were compared. The evaluation result of the VGG19FCN/B algorithm showed that the Dice similarity coefficient $(\mathrm{DSC})=0.9626$, mean pixel accuracy $(\mathrm{MPA})=0.9676$, and joint intersection $(\mathrm{IOU})=0.9155$, while the result of the $\mathrm{U}-\mathrm{Net} / \mathrm{B}$ algorithm was evaluated as $\mathrm{DSC}=0.9446$, $\mathrm{MPA}=0.9352$, and $\mathrm{IOU}=0.891$. The running time required for the VGG19-FCN was 13 times that of the U-Net model. Clinically, the segmentation of the left ventricle requires artificial judgment and analysis, which leads to low diagnosis efficiency and subjective judgment errors. Based on this, VGG19 in deep learning was adopted to design a model for ultrasonic cardiogram segmentation processing in this study. The results indicated that the VGG19-FCN and U-Net algorithm models based on the FCN proposed in this study can perform automatic left ventricular segmentation of the ultrasonic cardiogram, respectively, can meet the requirements of clinical applications, and can make up for the ultrasonic cardiogram. In addition, they can make up for the shortcomings of the ultrasonic cardiogram that is difficult to segment and judge in endocardial deletion.

\section{Conclusion}

This study aimed to use the deep learning technology to segment the ultrasonic cardiogram left ventricle and evaluate CHD to further enhance and improve the clinical diagnosis and treatment of CHD patients. The results showed that the VGG19-FCN and U-Net algorithm models can realize the segmentation processing of the ultrasonic cardiogram left ventricle, as well as the effective diagnosis and differentiation of $\mathrm{CHD}$ patients. In addition, the running time required for the VGG19-FCN was 13 times that of the U-Net model. Comprehensive analysis suggested that the U-Net model was more suitable for the practical application of automatic ultrasonic cardiogram segmentation. However, there were several shortcomings for this study. In the left ventricular segmentation model, only the spatial features of the heart were considered, the segmentation accuracy had to be improved, and the number of samples for patient evaluation was not large enough. The number of samples still needs to be expanded in the future, and different indicators should be evaluated more deeply. In summary, the study can effectively improve the efficiency of ultrasonic cardiogram left ventricular segmentation, show a significant effect on the diagnosis of CHD patients, and provide a reliable theoretical basis and foundation for the subsequent research of $\mathrm{CHD}$ imaging diagnosis.

\section{Data Availability}

The data used to support the findings of this study are available from the corresponding author upon request.

\section{Conflicts of Interest}

The authors declare no conflicts of interest.

\section{References}

[1] Y. Tian, P. Deng, B. Li et al., "Treatment models of cardiac rehabilitation in patients with coronary heart disease and related factors affecting patient compliance," Reviews in Cardiovascular Medicine, vol. 20, no. 1, pp. 27-33, 2019, PMID: 31184093.

[2] K. D. Boudoulas, F. Triposkiadis, P. Geleris, and H. Boudoulas, "Coronary atherosclerosis: pathophysiologic basis for diagnosis and management," Progress in Cardiovascular Diseases, vol. 58, no. 6, pp. 676-692, 2016, Epub 2016 Apr 25. PMID: 27091673.

[3] B. Cybulska and L. Kłosiewicz-Latoszek, "Landmark studies in coronary heart disease epidemiology. the framingham heart study after 70 years and the seven countries study after 60 years," Kardiologia Polska, vol. 77, no. 2, pp. 173-180, 2019, PMID: 30828782. 
[4] B. W. McCrindle and B. Cifra, "The role of echocardiography in kawasaki disease," International Journal of Rheumatic Diseases, vol. 21, no. 1, pp. 50-55, 2018, Epub 2017 Nov 19. PMID: 29152929.

[5] M. Kolossváry, C. N. De Cecco, G. Feuchtner, and P. Maurovich-Horvat, "Advanced atherosclerosis imaging by CT: radiomics, machine learning and deep learning," Journal of Cardiovascular Computed Tomography, vol. 13, no. 5, pp. 274-280, 2019, Epub 2019 Apr 21. PMID: 31029649.

[6] M. C. Williams, A. Hunter, A. Shah et al., "Symptoms and quality of life in patients with suspected angina undergoing CT coronary angiography: a randomised controlled trial," Heart, vol. 103, no. 13, pp. 995-1001, 2017, Epub 2017 Feb 28. PMID: 28246175; PMCID: PMC5529983.

[7] Á. Árnadóttir, K. R. Vestergaard, G. Sölétormos, R. Steffensen, J. P. Goetze, and K. Iversen, "Prediction of coronary heart disease or heart failure using high-sensitivity cardiac troponin T: a pilot study," European Journal of Clinical Investigation, vol. 48, no. 10, Epub 2018 Sep 6. PMID: 30062798, Article ID e13009, 2018.

[8] X. Jia, W. Sun, R. C. Hoogeveen et al., "High-sensitivity troponin i and incident coronary events, stroke, heart failure hospitalization, and mortality in the ARIC study," Circulation, vol. 139, no. 23, pp. 2642-2653, 2019, Epub 2019 Apr 29. PMID: 31030544; PMCID: PMC6546524.

[9] H. Baumgartner, J. Hung, J. Bermejo et al., "Recommendations on the echocardiographic assessment of aortic valve stenosis: a focused update from the European Association of Cardiovascular Imaging and the American Society of Echocardiography," European Heart Journal-Cardiovascular Imaging, vol. 18, no. 3, pp. 254-275, 2017, PMID: 28363204.

[10] M. Cameli, S. Mondillo, M. Galderisi et al., "L'ecocardiografia speckle tracking: roadmap per la misurazione e l'utilizzo clinico [Speckle tracking ultrasonic cardiogram a practical guide]," Glomale Italiano di Caradiologia, vol. 18, no. 4, pp. 253-269, 2017, PMID: 28492566.

[11] H. Baumgartner, J. Hung, J. Bermejo et al., "Recommendations on the echocardiographic assessment of aortic valve stenosis: a focused update from the european association of cardiovascular imaging and the american society of echocardiography," Journal of the American Society of Echocardiography, vol. 30, no. 4, pp. 372-392, 2017, PMID: 28385280.

[12] J. Hung, S. L. Klassen, J. Bermejo, and J. B. Chambers, "Take home messages with cases from focused update on echocardiographic assessment of aortic stenosis," Heart, vol. 104, no. 16, pp. 1317-1322, 2018, Epub 2018 Mar 23. PMID: 29572249.

[13] I. Stojanovic, J. E. Schneider, and J. Cooper, "Cost-impact of cardiac magnetic resonance imaging with Fast-SENC compared to SPECT in the diagnosis of coronary artery disease in the U.S," Journal of Medical Economics, vol. 22, no. 5, pp. 430-438, 2019, Epub 2019 Mar 15. PMID: 30732489.

[14] E. Kazakauskaitè, D. Žaliaduonytè-Pekšienè, E. Rumbinaite, J. Keršulis, I. Kulakienè, and R. Jurkevičius, "Positron emission tomography in the diagnosis and management of coronary artery disease," Medicina, vol. 54, no. 3, p. 47, 2018 PMID: 30344278; PMCID: PMC6122121.

[15] H. Xue, E. Tseng, K. D. Knott et al., "Automated detection of left ventricle in arterial input function images for inline perfusion mapping using deep learning: a study of 15,000 patients," Magnetic Resonance in Medicine, vol. 84, no. 5, pp. 2788-2800, 2020, Epub 2020 May 7. PMID: 32378776.

[16] X. Q. Lao, X. Liu, H.-B. Deng et al., "Sleep quality, sleep duration, and the risk of coronary heart disease: a prospective cohort study with 60,586 adults," Journal of Clinical Sleep Medicine, vol. 14, no. 1, pp. 109-117, 2018, PMID: 29198294; PMCID: PMC5734879.

[17] I. Danad, J. Szymonifka, J. W. R. Twisk et al., "Diagnostic performance of cardiac imaging methods to diagnose ischaemia-causing coronary artery disease when directly compared with fractional flow reserve as a reference standard: a meta-analysis," European Heart Journal, vol. 38, no. 13, pp. ehw095-998, 2017, PMID: 27141095; PMCID: PMC5381594.

[18] I. Mordi, A. Badar, R. Irving, J. Weir-McCall, G. Houston, and C. Lang, "Efficacy of noninvasive cardiac imaging tests in diagnosis and management of stable coronary artery disease," Vascular Health and Risk Management, vol. 13, pp. 427-437, 2017, PMID: 29200864; PMCID: PMC5701553.

[19] L. Qiu, H. Tan, D. Cheng, and H. Shi, "The incremental clinical value of cardiac hybrid SPECT/CTA imaging in coronary artery disease," Nuclear Medicine Communications, vol. 39, no. 6, pp. 469-478, 2018, PMID: 29652747.

[20] L. Faroux, L. Guimaraes, J. Wintzer-Wehekind et al., "Coronary artery disease and transcatheter aortic valve replacement," Journal of the American College of Cardiology, vol. 74, no. 3, pp. 362-372, 2019, PMID: 31319919.

[21] A. Mangla, E. Oliveros, K. A. Williams Sr, and D. K. Kalra, "Cardiac imaging in the diagnosis of coronary artery disease," Current Problems in Cardiology, vol. 42, no. 10, pp. 316-366, 2017, Epub 2017 Apr 12. PMID: 28870377. 